\title{
Forkhead box N1 inhibits the progression of non-small cell lung cancer and serves as a tumor suppressor
}

\author{
XIAOJIAN JI ${ }^{1}$, YAN JI ${ }^{2}$, WENQING WANG ${ }^{1}$ and XINMEI XU ${ }^{1}$ \\ ${ }^{1}$ Emergency Department, The People's Hospital of Guizhou Province, Guiyang, Guizhou 550002, \\ P.R. China; ${ }^{2}$ Department of Hematology and Oncology, Regions Hospital, St. Paul, MN 55101, USA
}

Received April 7, 2017; Accepted November 10, 2017

DOI: $10.3892 /$ ol.2018.8210

\begin{abstract}
Forkhead box N1 (FOXN1) belongs to the FOX family of transcription factors, which comprises a diverse group of winged-helix proteins. FOXN1 is a ubiquitously expressed member that has been implicated in the embryo development, metabolism, aging and cancer. However, little is known regarding the role of FOXN1 in non-small cell lung cancer (NSCLC). The aim of the study was to investigate the function of FOXN1 in NSCLC and examine the relevant mechanism. In the present study, using reverse transcription-quantitative polymerase chain reaction, western blotting, transwell assay, MTT assay, luciferase report assy, it was identified that knockdown of FOXN1 increased the proliferation of A549 and H1299 cells, while overexpression of FOXN1 evidently suppressed the cell growth. A Transwell assay was used to determine the relative cell invasion ability, and it was observed that the invading cells were markedly decreased in the FOXN1 overexpression groups; by contrast, reduced expression of FOXN1 demonstrated the potential to promote cell invasion. Furthermore, lower expression of FOXN1 was observed in NSCLC tissues and cell lines as compared with the adjacent non-tumor tissues or human bronchial epithelial cells, respectively. A higher level of FOXN1 was associated with a better prognosis of NSCLC patients. Quantitative chromatin immunoprecipitation analysis and luciferase reporter gene assays revealed that enhancer of zeste homolog 2 (EZH2) and $\beta$-catenin were two target genes of FOXN1. Reverse transcription-quantitative polymerase chain reaction (RT-qPCR) and western blot analysis indicated that FOXN1 suppressed the expression levels of these target genes at the transcriptional level. In conclusion, the present study demonstrated that FOXN1 served major roles in NSCLC proliferation and invasion by directly repressing EZH2 and
\end{abstract}

Correspondence to: Dr Xiaojian Ji, Emergency Department, The People's Hospital of Guizhou Province, 83 East Zhongshan Road, Guiyang, Guizhou 550002, P.R. China

E-mail: zpjrvh@163.com

Key words: forkhead box N1, non-small cell lung cancer, proliferation, invasion, enhancer of zeste homolog $2, \beta$-catenin $\beta$-catenin, which suggested that FOXN1 may function as a tumor suppressor in NSCLC.

\section{Introduction}

Lung cancer is one of the most common types of cancer and the leading cause of cancer-associated mortality worldwide $(1,2)$. Non-small cell lung cancer (NSCLC) is the main type of lung cancer and accounts for nearly $84 \%$ of cases. Despite decades of intensive efforts in the cancer research field, the prognosis of patients with NSCLC remains poor, with a 5-year overall survival rate of $\sim 10 \%$ (3). Thus, further investigation of novel biomarkers and therapeutic targets for NSCLC is urgently required (4).

The family of forkhead box (FOX) transcription factors, which includes the FOXP subfamily, serves important roles in oncogenesis. Notably, downregulation of FOXP4 markedly reduced the NSCLC cell growth and invasion (5), while FOXP2 worked as a potential novel tumor metastasis suppressor in breast cancer (6). In NSCLC, higher expression of FOXN1 has been associated with improved survival times in patients (7). Using tissue arrays and immunohistochemical analysis, FOXP2 has been observed to function as a novel candidate tumor suppressor gene in different cancer types. However, to our knowledge, the function of FOXN1 in cancer remains unknown. In the present study, the aim was to evaluate the role of FOXN1 in NSCLC.

EZH2 is overexpressed and associated with worse overall survival rate in NSCLC patients (8). Nuclear $\beta$-catenin could serves as an important prognosticator for patients with NSCLC (9).

\section{Materials and methods}

Cell lines and reagents. Normal human bronchial epithelial (HBE) cells, A549, H1299, SPC-A1, H460, H520 and SK-MES-1 lung cancer cell lines were purchased from the American Type Culture Collection (Manassas, VA, USA). The cells were cultured at $37^{\circ} \mathrm{C}$ in a humidified atmosphere with $5 \% \mathrm{CO}_{2}$ in RPMI-1640 medium (Invitrogen; Thermo Fisher Scientific, Inc., Waltham, MA, USA) supplemented with $10 \%$ fetal bovine serum (FBS; Hyclone; Thermo Fisher Scientific, Inc.) and $1 \%$ penicillin/streptomycin (Invitrogen; Thermo Fisher Scientific, Inc.). Mouse anti-human FOXN1 polyclonal 
antibody (cat no. sc-271256; 1:500), rabbit anti-human enhancer of zeste homolog 2 (EZH2) polyclonal antibody (cat no. sc-25383; 1:1,000), mouse anti-human $\beta$-actin monoclonal antibody (cat. no. SC47778; 1:2,000) horseradish peroxidase-labeled secondary antibodies including goat anti-mouse IgG-horseradish peroxidase (cat no. sc-2005; 1:3,000) and anti-rabbit IgG-horseradish peroxidase (cat no. sc-2004; 1:3,000) were purchased from Santa Cruz Biotechnology, Inc. (Dallas, TX, USA).

Transfection for FOXN1 downregulation. Two different FOXN1-short hairpin RNA (shRNA) ShFOXN1\#1 TRCN0000013197 (CCGGCCTCCTGCTATGGGCAG ACATCTCGAGATGTCTGCCCATAGCAGGAGGTTTTT) ShFOXN1\#2 TRCN0000013193 (CCGGGCTCACACTCA TCCACACTTACTCGAGTAAGTGTGGATGAGTGTGAGC TTTTT) ShEZH2 TRCN0000040074 (CCGGGCTAGGTT AATTGGGACCAAACTCGAGTTTGGTCCCAATTAACC TAGCTTTTTG) Sh $\beta$-catenin TRCN0000314991 (CCGGTT GTTATCAGAGGACTAAATACTCGAGTATTTAGTCCTC TGATAACAATTTTTG) or non-targeting negative control shRNA was designed and synthesized by Sigma-Aldrich (Merck KGaA; Darmstadt, Germany).

Transfection for FOXN1 overexpression. pcDNA3.1-FOXN1 was cloned by GenePharm Co., Ltd. (Shanghai, China). pcDNA3.1 was used the control. Cultured cells were subjected to transfection using Lipofectamine ${ }^{\circledR} 3000$ (Invitrogen; Thermo Fisher Scientific, Inc.), according to the manufacturer's protocol. A total of $20 \mu \mathrm{M}$ relative shRNA or $5 \mu \mathrm{g}$ relative plasmid were used for transfection. Following incubation at $37^{\circ} \mathrm{C}$ for $48-72 \mathrm{~h}$, cells were collected and lysed to verify the expression of target genes or proteins by reverse transcription-quantitative polymerase chain reaction (RT-qPCR) and western blot analysis, respectively.

Patients. A total of 60 NSCLC patients diagnosed and treated at the People's Hospital of Guizhou Province (Guiyang, China) were enrolled into the present study. Written informed consent was obtained from each patient, and the current study was approved by the Ethics Committee of the People's Hospital of Guizhou Province. Tumor tissues and the adjacent normal tissues were attained during surgery between February 2010 and December 2014. Tissue samples were frozen and stored at $-80^{\circ} \mathrm{C}$ for RT-PCR. Histological and pathological diagnoses were performed by the pathology department. The tissues were embedded in paraffin and cut into $2 \mu \mathrm{m}$ sections. Slides were incubated in three washes of xylene for $5 \mathrm{~min}$ each, followed by two washes of $100 \%$ ethanol for $10 \mathrm{~min}, 95 \%$ ethanol for $10 \mathrm{~min}$ and $\mathrm{ddH}_{2} \mathrm{O}$ for $5 \mathrm{~min}$. Then the samples were blocked in $10 \%$ normal goat serum in PBS at room temperature for $30 \mathrm{~min}$ and incubated at $4^{\circ} \mathrm{C}$ overnight in primary antibody solution of anti-FOXN1 (1:100) The EnVision Detection System kit (Dako; Agilent Technologies, Inc., Santa Clara, CA, USA) was used to visualize the 3,3'-diaminobenzidine chromogen (room temperature for $20 \mathrm{~min}$ ). Samples were counterstained with hematoxylin $(0.2 \%)$ at room temperature for 5 min (Zhongshan Golden Bridge Biotechnology Company) according to the Staging Lung Cancer's Revised
International System (10). Neutral gum was used to cover the slides and they were dried at room temperature. Staining was visualized under an Olympus optical microscope (Olympus Corporation, Tokyo, Japan) at x20 magnification for analysis.

Western blot analysis. Lung cancer cells were harvested and lysed using $0.5 \%$ NP-40 cell lysis buffer (Sigma-Aldrich; Merck $\mathrm{KGaA}$ ) for $20 \mathrm{~min}$ on ice, and transparent protein lysates were obtained following centrifugation at 12,000 $\mathrm{x}$ g for $10 \mathrm{~min}$ at $4^{\circ} \mathrm{C}$. The Bradford assay reagent (Thermo Fisher Scientific, Inc.) was then used to determine the protein concentration in the lysates. Next, $30 \mu \mathrm{g}$ protein from each group was separated by $10 \%$ SDS-PAGE and then transferred onto polyvinylidene difluoride membranes. Subsequently, $5 \%$ skim milk was used to block nonspecific binding in the samples at room temperature for $1 \mathrm{~h}$, followed by addition of the primary antibodies against FOXN1, EZH2 and $\beta$-actin for overnight incubation at $4^{\circ} \mathrm{C}$. $\beta$-actin served as the internal control. The membranes were then washed with Tris-buffered saline/Tween 20 for $5 \mathrm{~min}$ and five times, incubated with respective secondary antibodies goat anti-mouse IgG-horseradish peroxidase $(1: 3,000)$ and anti-rabbit IgG-horseradish peroxidase $(1: 3,000)$ at room temperature for $1 \mathrm{~h}$ and visualized using an enhanced chemiluminescence-based methods (western blotting detection system; Thermo Fisher Scientific, Inc.).

$R N A$ extraction and $R T-q P C R$. Total RNA was extracted from the tissues or cells using TRIzol reagent (Invitrogen; Thermo Fisher Scientific, Inc.), according to the manufacturer's protocol. The RNA concentration was assessed using a nanophotometer UV/VIS spectrophotometer (Implen GmbH, München, Germany). A total of $2 \mu \mathrm{g}$ RNA was converted into cDNA using Revert Aid ${ }^{\mathrm{TM}}$ First Strand cDNA Synthesis kit (Fermentas; Thermo Fisher Scientific, Inc.). The qPCR reaction was subsequently performed on a 7500 Real-Time Detection System (Applied Biosystems; Thermo Fisher Scientific, Inc.) according to the following conditions: Initial step, $95^{\circ} \mathrm{C}$ for $3 \mathrm{~min}$; second step, $95^{\circ} \mathrm{C}$ for $10 \mathrm{sec}, 60^{\circ} \mathrm{C}$ for $30 \mathrm{sec}$ and $72^{\circ} \mathrm{C}$ for $10 \mathrm{sec}$ for a total of 38 cycles. The obtained data were analyzed using the $2^{-\Delta \Delta C a}$ method, (11) with GAPDH used as an internal control. The primers used were as follows: GAPDH, forward 5'-GAGAAGTATGACAACAGCCTC-3' and reverse 5'-ATGGACTGTGGTCATGAGTC-3'; FOXN1, forward 5'-GCTCCTCACACTATCAGTACC-3' and reverse 5'-AAGATGAGGATGCTGTAGGA-3'.

Invasionassay.Transwellmigrationchambers (BDBiosciences, Franklin Lakes, NJ, USA) were used to measure the invasive capacity of cells. Briefly, $100 \mu 1$ Matrigel (BD Biosciences) was coated onto $8-\mu \mathrm{m}$ filter chambers and plated on 24-well plates. A total of $2 \times 10^{4}$ cells were resuspended using $100 \mu \mathrm{l}$ Dulbecco's modified Eagle's medium (DMEM; Invitrogen; Thermo Fisher Scientific, Inc.) basal medium without FBS and seeded into the upper chamber, while $500 \mu \mathrm{l}$ DMEM with $10 \%$ FBS was added into the lower chamber. Following incubation at $37^{\circ} \mathrm{C}$ in a $5 \% \mathrm{CO}_{2}$ atmosphere for $12 \mathrm{~h}$, the medium was removed and the cells were washed twice with cool PBS to remove cells from the upper chamber. Subsequent to fixing with $4 \%$ paraformaldehyde for $15 \mathrm{~min}$, the invading cells in the lower chamber were stained with $1 \%$ crystal violet for 

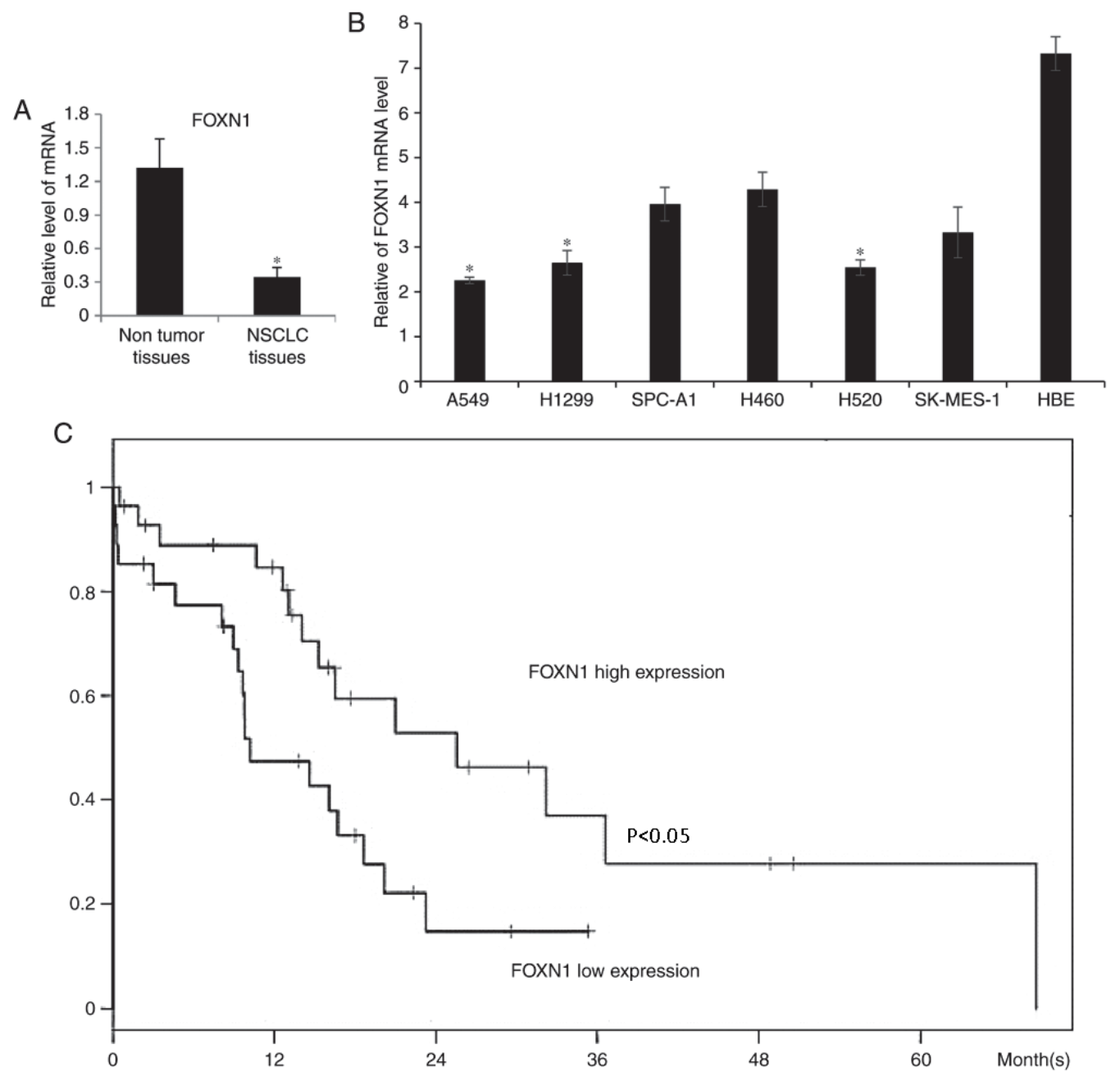

Figure 1. Increased expression of FOXN1 is associated with improved prognosis of NSCLC patients. (A) NSCLC tissues and paired noncancerous lung tissues ( $\mathrm{n}=60$ ), as well as (B) NSCLC cell lines (A549, H1299, SPC-A1, H460, H520 and SK-MES-1) and normal HBE cells, were examined by reverse transcription-quantitative polymerase chain reaction to measure the FOXN1 mRNA levels. ${ }^{*} \mathrm{P}<0.05$ vs. HBE cells. (C) Kaplan-Meier curves were constructed to measure the patient survival rate, and Cox log-rank test was used to measure the prognostic significance. The $\mathrm{x}$-axis represents the survival months, while the $\mathrm{y}$-axis represents the survival probability. $(\mathrm{P}<0.05)$. FOXN1, forkhead box N1; NSCLC, non-small cell lung cancer; HBE, human bronchial epithelial cells.

$15 \mathrm{~min}$. The number of cells was counted under an upright light microscope in 10 different fields of view (Leica DM4B; Leica Microsystems, Shanghai, China). At least three independent experiments were performed.

MTT assay. Cell proliferation was determined using an MTT proliferation kit (Beijing Solarbio Science \& Technology Co., Ltd., Beijing, China). The transfected cells were subjected to centrifugation at $800 \mathrm{x} \mathrm{g}$ for $5 \mathrm{~min}$ at $4^{\circ} \mathrm{C}$, followed by resuspension with complete medium. Next, the cells were seeded into a 96 -well plate at a density of $2 \times 10^{4}$ cells per well. Following incubation for 24, 48 and 72 h, MTT reagent was added and an MTT assay was performed following the manufacturer's protocol. Each group was examined in three separate wells.

Quantitative chromatin immunoprecipitation (qChIP) analysis. The qChIP assay was performed using the Chip-IT Express kit (Active Motif, Carlsbad, CA, USA), according to the manufacturer's protocol. qPCR was performed with $5 \mu 1$ of the immunoprecipitated target DNA, $1 \mu 1$ primers and $9 \mu 1$ mixture (1 $\mu 1$ enzyme, $2 \mu \mathrm{l}$ dNTP and $6 \mu 1 \mathrm{SYBR}$ green solution buffer; all included in the ChIP-IT kit). The primers used were as follows: EZH2, forward, 5'-GAGGCA TGAGAATCGCTTGA-3', and reverse, 5'-GCCGGACCC GTTACTACTTT-3'; $\beta$-catenin, forward, 5'-CAGTTGGCA TTACCACTTAT-3', and reverse, 5'-TTCACTCATGGAGGT AGGAT-3'. The PCR amplification was performed at $95^{\circ} \mathrm{C}$ for $5 \mathrm{~min}$, followed by 35 cycles of $95^{\circ} \mathrm{C}$ for $20 \mathrm{sec}, 55^{\circ} \mathrm{C}$ for $20 \mathrm{sec}$ and $72^{\circ} \mathrm{C}$ for $30 \mathrm{sec}$. The $2^{-\Delta \Delta \mathrm{Cq}}$ method of quantification was used, with GAPDH used as an internal control, as per the RT-qPCR section.

Dual-luciferase reporter assay. A dual-luciferase reporter gene system (Promega Corporation, Madison, WI, USA) was used to verify the direct target genes for FOXN1, according to the manufacturer's protocol. Briefly, the full length of EZH2 promoter was synthesized by clonal expansion and cloned into the pmirGLO luciferase gene vector (Promega Corporation). FOXN1 and control plasmids were co-transfected into the cells along with the luciferase reporter vectors using Lipofectamine 2000 (Invitrogen; Thermo Fisher 
Table I. Clinicopathological variables in 60 non-small cell lung cancer patients.

\begin{tabular}{|c|c|c|c|c|}
\hline \multirow[b]{2}{*}{ Variables } & \multirow[b]{2}{*}{ Patient no. $(\mathrm{n}=60)$} & \multicolumn{2}{|c|}{ FOXN1 protein expression } & \multirow[b]{2}{*}{ P-value } \\
\hline & & Low $(\mathrm{n}=37)$ & High $(n=23)$ & \\
\hline Age (years) & & & & 0.887 \\
\hline$<50$ & 32 & 20 & 12 & \\
\hline$\geq 50$ & 28 & 17 & 11 & \\
\hline Gender & & & & 0.210 \\
\hline Male & 33 & 18 & 15 & \\
\hline Female & 27 & 19 & 8 & \\
\hline Tumor size & & & & 0.017 \\
\hline Small $(\leq 3 \mathrm{~cm})$ & 30 & 14 & 16 & \\
\hline Large $(\geq 3 \mathrm{~cm})$ & 30 & 23 & 7 & \\
\hline Metastasis & & & & 0.002 \\
\hline Present & 36 & 28 & 8 & \\
\hline Absent & 24 & 9 & 15 & \\
\hline TNM stage & & & & $<0.001$ \\
\hline $\mathrm{I}+\mathrm{II}$ & 28 & 10 & 18 & \\
\hline III + IV & 32 & 27 & 5 & \\
\hline Differentiation & & & & 0.580 \\
\hline Well/moderate & 34 & 22 & 12 & \\
\hline Poor & 26 & 15 & 11 & \\
\hline
\end{tabular}

Scientific, Inc.). After $36 \mathrm{~h}$ at $37^{\circ} \mathrm{C}$, the expression of Renilla (Takara Biotechnology Co., Ltd., Dalian, China) was used as an internal reference to adjust the number of cells and the transfection efficiency differences.

Statistical analysis. All experiments were performed at least three times. The data were analyzed using SPSS version 17.0 (SPSS, Inc., Chicago, IL, USA). Data was expressed as the mean \pm standard deviation of three independent experiments. Differences between the experimental and control groups were compared using Student's t-test to compare differences between two groups. A two-way analysis of variance was performed in cases where more than two groups were compared. The post hoc test employed was Tukey's range test. $\mathrm{P}<0.05$ was considered to indicate a statistically significant difference.

\section{Results}

FOXN1 is downregulated in NSCLC tissues and cells. In order to understand the role of FOXN1 in NSCLC, 60 pairs of tissue samples were collected from NSCLC patients, and the mRNA expression of FOXN1 was determined by RT-qPCR. As illustrated in Fig. 1A, the expression of FOXN1 was significantly downregulated in tumor tissues when compared with the paired noncancerous tissues $(\mathrm{P}<0.05)$. Furthermore, FOXN1 mRNA expression was detected in six NSCLC cell lines, namely A549, H1299, SPC-A1, H460, H520 and SK-MES-1, and normal human bronchial epithelial (HBE) cells. It was observed that the mRNA level of FOXN1 was lower in the NSCLC cell lines, with a significant effect observed for A549,
H1299 and H520 cells, as compared with the HBE cells (P<0.05; Fig. 1B). A549 and H1299 cells were used for subsequent experiments.

Higher expression of FOXN1 is associated with better prognosis in NSCLC patients. To further determine the function of FOXN1 during NSCLC development and progression, the association of FOXN1 expression with the NSCLC patient clinicopathological characteristics, including the gender, age, tumor size, differentiation, metastasis and TNM staging, was analyzed. As shown in Table I, a higher expression of FOXN1 was associated with smaller tumor size, absence of metastasis and lower TNM stage $(\mathrm{P}<0.05)$, which indicated an improved prognosis. Furthermore, Kaplan-Meier curves were constructed to analyze the NSCLC patient survival rate. As shown in Fig. 1C, a higher FOXN1 expression was correlated with a better overall survival rate $(\mathrm{P}<0.05)$.

FOXN1 suppresses NSCLC cell proliferation. In order to determine the role of FOXN1 in NSCLC cells, the effect of FOXN1 overexpression on A549 and H1299 cell proliferation was investigated. Initially, two specific shRNAs targeted against FOXN1 (namely shFOXN1-1 and shFOXN1-2) were used to reduce the expression of FOXN1 in NSCLC cells. In addition, stable A549 or H1299 cell lines with overexpression of FOXN1 were established by plasmid transfection. The cells transfected with pcDNA3.1 plasmid was named as vector group, while the cells transfected with FOXN1 plasmid was named as FOXN1. The successful knockdown (Fig. 2A) and overexpression (Fig. 2B) of FOXN1 in the cells was confirmed by RT-qPCR. Subsequently, an MTT assay 
A
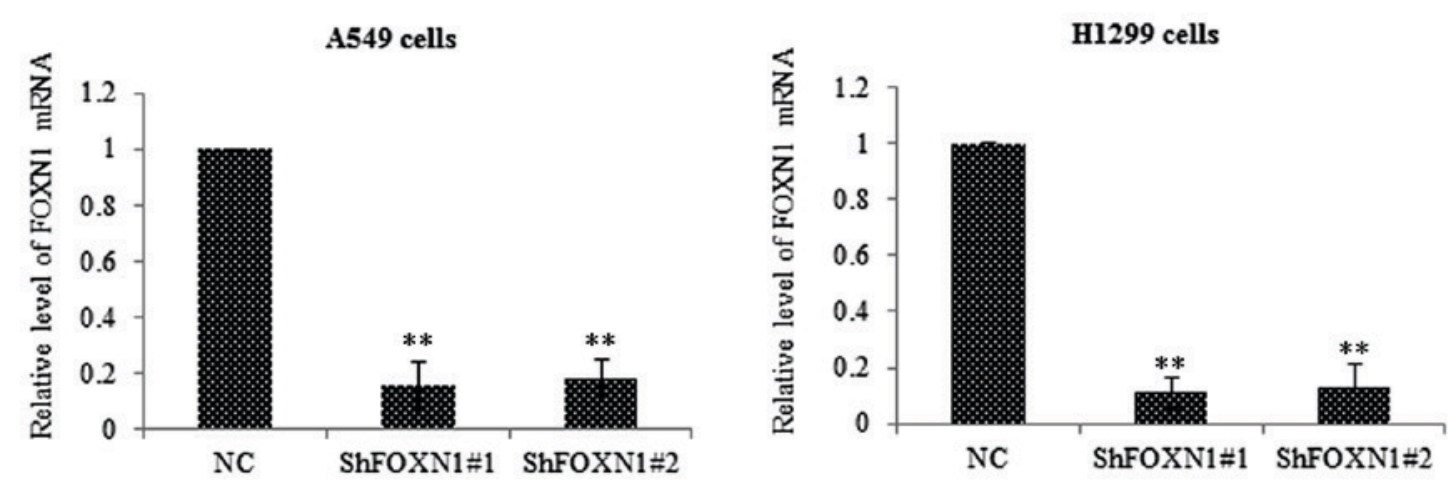

B
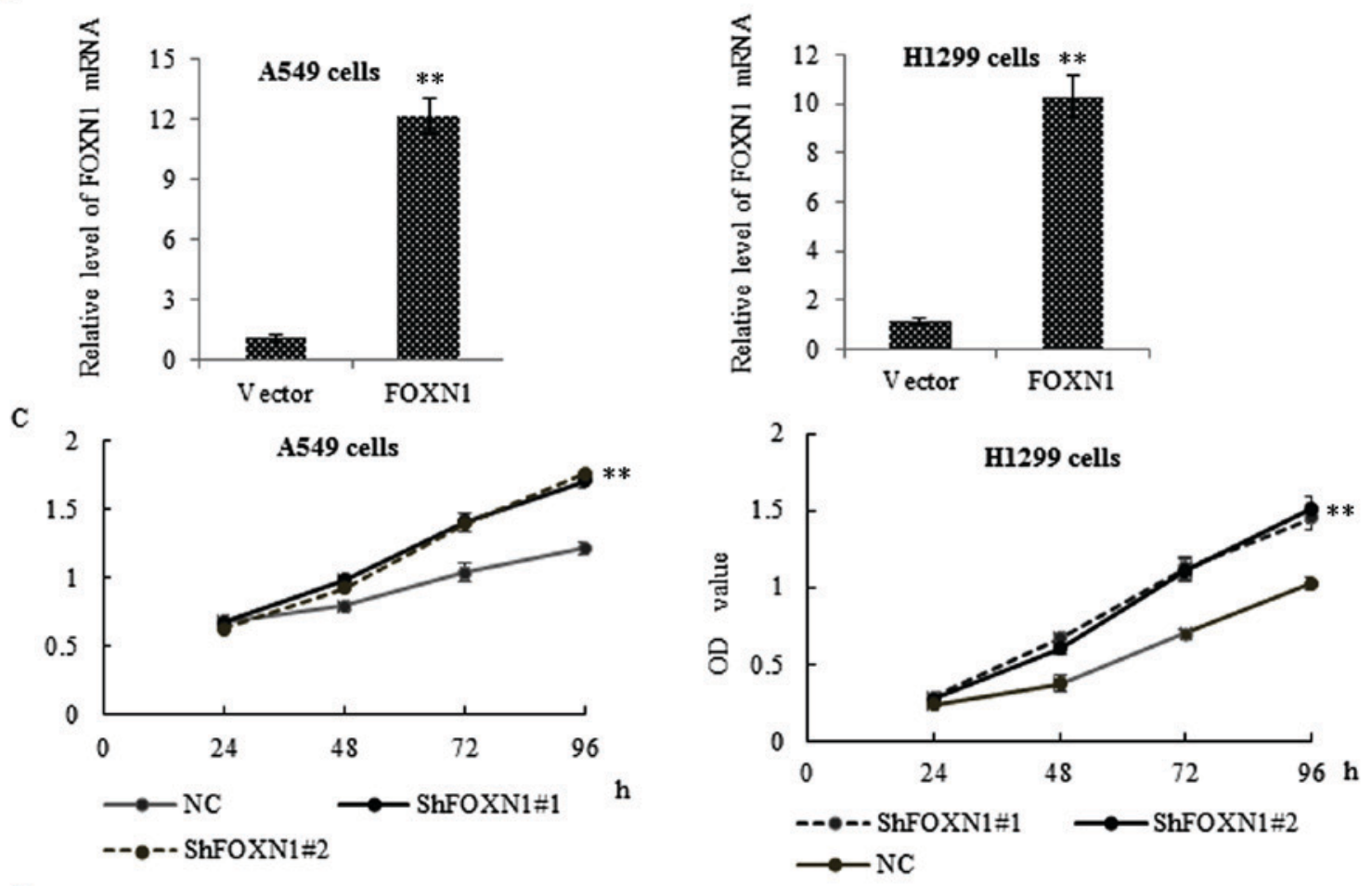

D
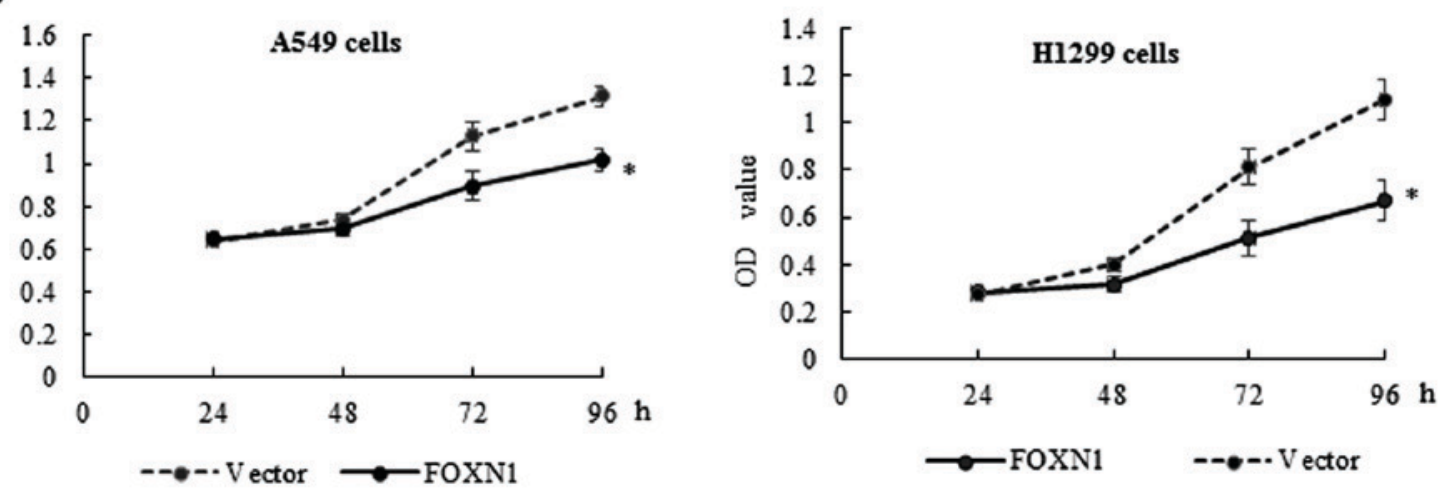

Figure 2. FOXN1 suppresses non-small cell lung cancer cell proliferation. Reverse transcription-quantitative polymerase chain reaction was performed to measure the FOXN1 mRNA expression in A549 or H1299 cells transfected with (A) shRNA targeting FOXN1 or NC shRNA, and (B) a lentivirus expressing FOXN1 or with a vector. The relative FOXN1 mRNA level was normalized against GAPDH expression. Furthermore, an MTT assay was conducted to determine the proliferation of A549 or H1299 cells transfected with (C) shRNA targeting FOXN1 or NC shRNA, and (D) a lentivirus expressing FOXN1 or vector. The results were detected at 24,48 and $72 \mathrm{~h}$. Values are expressed as the mean \pm standard deviation of three independent measurements. ${ }^{*} \mathrm{P}<0.05$ and ${ }^{* *} \mathrm{P}<0.01$, vs. corresponding control group. FOXN1, forkhead box N1; shRNA, short hairpin RNA; NC, negative control shRNA; OD, optical density.

was performed, which revealed that the cell growth was significantly promoted in cells transfected with shFOXN1 for expression knockdown as compared with the control cells
(Fig. 2C). By contrast, ectopic overexpression of FOXN1 in the A549 and H1299 cells markedly suppressed the cell growth (Fig. 2D). 

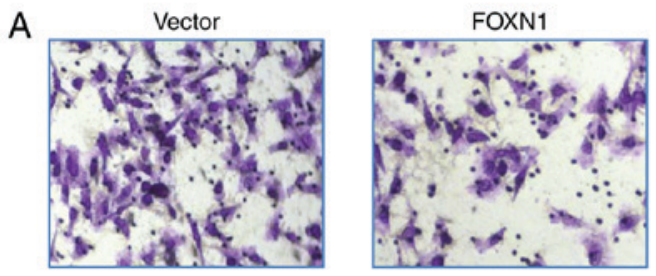

B
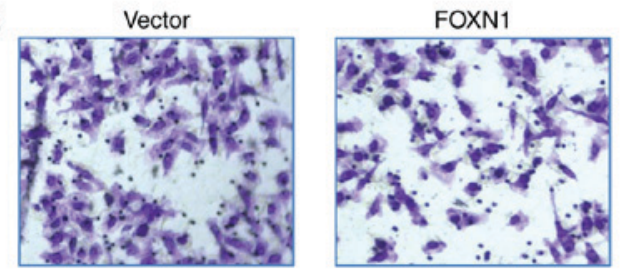

C

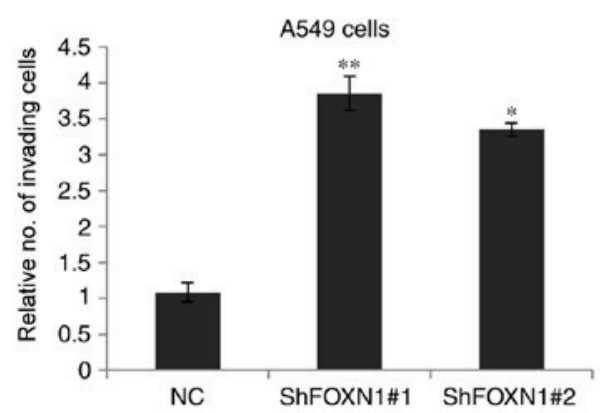

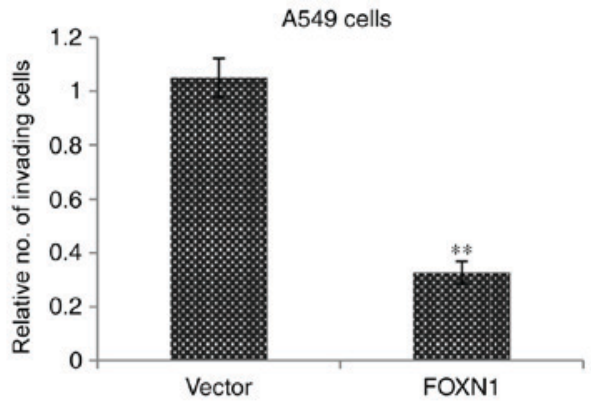

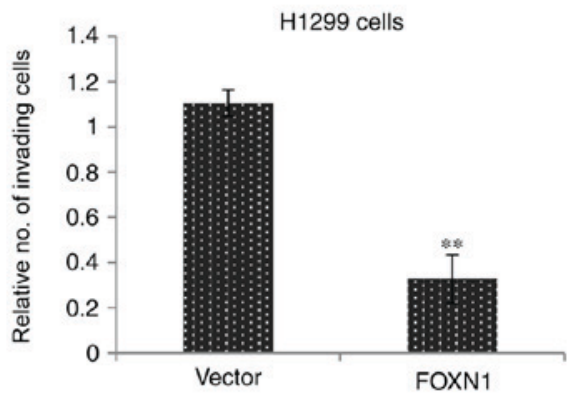

D

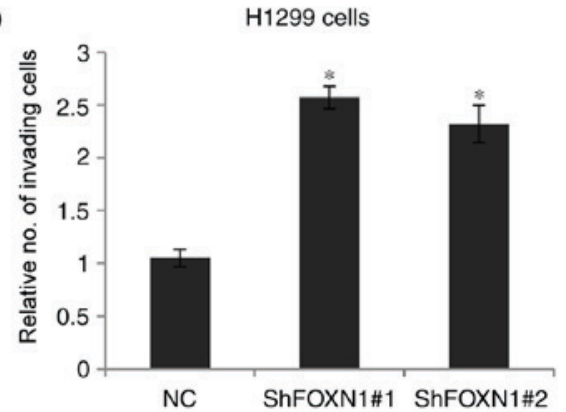

Figure 3. FOXN1 inhibits non-small cell lung cancer cell invasion, as determined by a Transwell assay. (A) A549 or (B) H1299 cells were transfected for $48 \mathrm{~h}$ with lentivirus expressing FOXN1 to induce FOXN1 overexpression or a control vector. Images are of cells stained with $1 \%$ crystal violet staining, 20x magnification. (C) A549 or (D) H1299 cells were transfected for 24 h with shRNA targeting FOXN1 to inhibit FOXN1 expression or NC shRNA. The number of invading cells was expressed as the mean \pm standard deviation of three independent experiments. ${ }^{*} \mathrm{P}<0.05$ and ${ }^{* *} \mathrm{P}<0.01$, vs. corresponding control group. FOXN1, forkhead box N1; shRNA, short hairpin RNA; NC, negative control shRNA.

FOXN1 inhibits NSCLC cell invasion. To examine whether FOXN1 exerts an effect on the A549 and H1299 cell invasive ability, a Transwell assay was performed. In this assay, the number of cells that migrated from the upper to the lower side of the membrane in the chambers was calculated. As shown in Fig. 3A and B, the number of invading cells was markedly decreased in the FOXN1 overexpression plasmid-transfected cells as compared with the control group. By contrast, a reduced expression of FOXN1 demonstrated the potential to promote cell invasion in A549 (Fig. 3C) and H1299 cells (Fig. 3D), respectively. These results suggested that the overexpression of FOXN1 clearly suppressed the invasion ability of A549 and H1299 cells.

EZH2 and $\beta$-catenin are novel target genes of FOXN1. In order to understand the underlying mechanisms of FOXN1 in suppressing NSCLC, a quantitative chromatin immunoprecipitation (ChIP) assay was performed in A549 and H1299 cells, with normal IgG used as a negative control. Different genes involved in proliferation and invasion were selected for investigation, including E-cadherin, $\mathrm{N}$-cadherin, r-catenin, $\beta$-catenin, caspase-3, caspase-7, hypoxia-inducible factor $1 \alpha$ (HIF1A), EZH2, Snail and Twist1. As shown in
Fig. 4A, in A549 cells, the bindings of FOXN1 to E-cadherin and $\beta$-catenin promoters was were significantly higher when compared with that of the normal IgG. Similar binding enrichment was also detected in H1299 cells (Fig. 4B). FOXN1 is known to typically function as a transcription repressor factor (12); thus, to further support this observation, a dual-luciferase reporter assay was conducted in A549 and H1299 cells. Cells co-transfected with shFOXN1 along with EZH2 or $\beta$-catenin promoter-driven luciferase reporters exhibited a moderately enhanced luciferase activity, as compared with cells transfected with Renilla and pcDNA3.1 plasmids (Fig. 4C). By contrast, in the FOXN1 overexpression group, reduced luciferase activity was detected (Fig. 4D).

Inhibition of the EZH2/ $\beta$-catenin cascade is involved in the suppressive effect of FOXN1 on NSCLC. Consistent with the results of the dual-luciferase reporter assay, in A459 and H1299 cells subjected to FOXN1 knockdown, significantly increased mRNA levels of EZH2 and $\beta$-catenin were confirmed by RT-qPCR assay. By contrast, ectopic overexpression of FOXN1 in A549 and H1299 cells markedly suppressed the EZH2 and $\beta$-catenin mRNA levels (Fig. 5A). Furthermore, as 

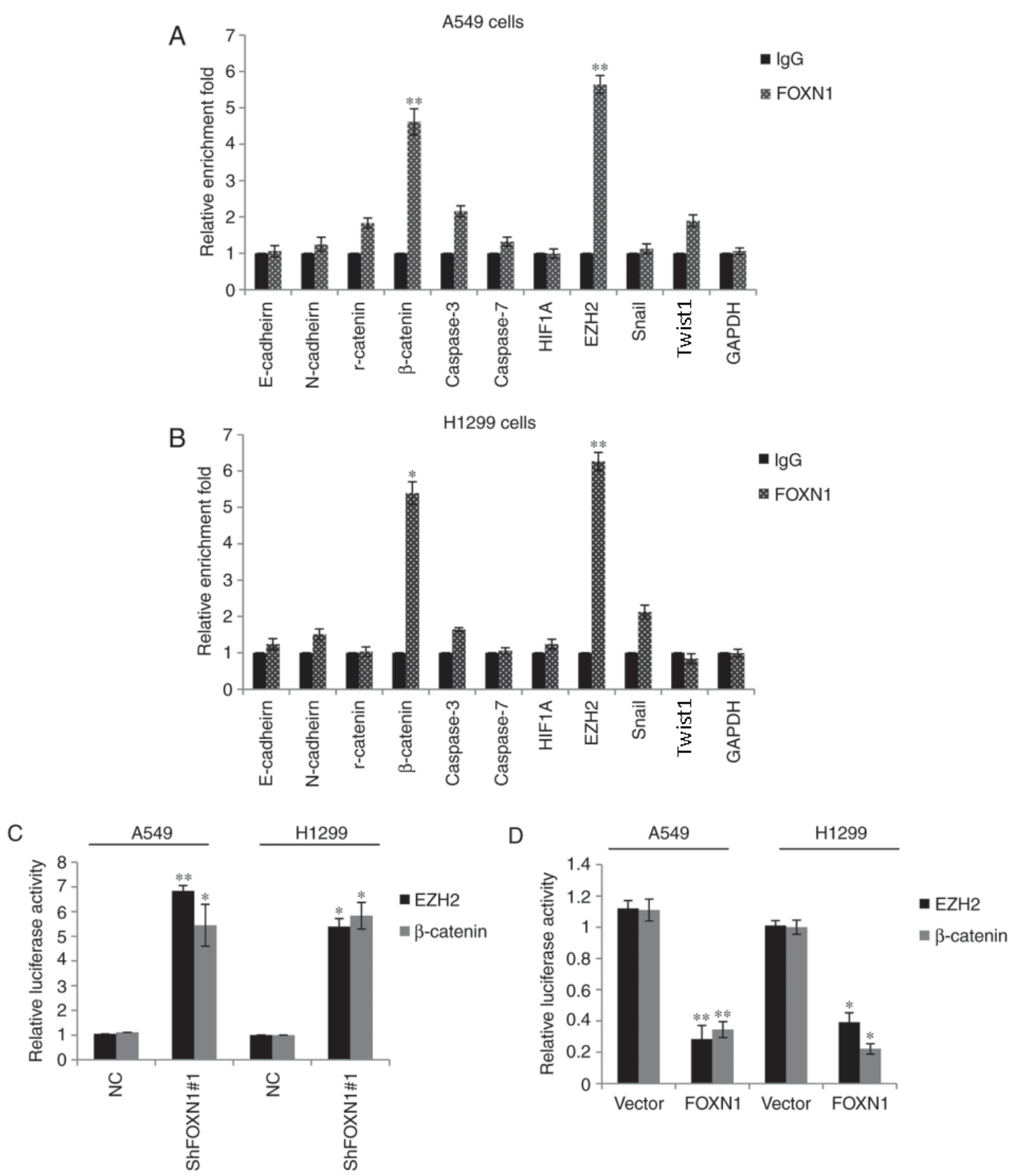

Figure 4. EZH2 and $\beta$-catenin are novel target genes of FOXN1. Quantitative chromatin immunoprecipitation experiment was performed in (A) A549 or (B) H1299 cells using an antibody against FOXN1 or normal IgG (negative control). The binding of FOXN1 on EZH2 and $\beta$-catenin promoters were measured. A549 or H1299 cells were transfected with EZH2 or $\beta$-catenin promoter luciferase constructs along with (C) FOXN1 silencing molecules or NC shRNA, or (D) FOXN1 overexpression construct or pcDNA3.1 plasmids. The luciferase activities were measured and normalized to those of Renilla. Values are represented as the mean \pm standard deviation of three independent experiments. ${ }^{*} \mathrm{P}<0.05$ and ${ }^{* * *} \mathrm{P}<0.01$, vs. corresponding control group. FOXN1, forkhead box N1; shRNA, short hairpin RNA; NC, negative control shRNA; EZH2, enhancer of zeste homolog 2.

shown in Fig. 5B, shFOXN1 transfection increased the protein levels of EZH2 and $\beta$-catenin, while overexpression of FOXN1 decreased the protein levels of EZH2 and $\beta$-catenin.

To establish further insight into the mechanism, the study subsequently examined whether EZH2 and $\beta$-catenin were involved in the suppressive effect of FOXN1 on NSCLC cell proliferation and invasion. An MTT assay was performed, and revealed that the additional knockdown of EZH2 or $\beta$-catenin by shRNA transfection was observed to partially rescue the effect of FOXN1 knockdown on the proliferation potential of A549 and H1299 cells, as compared with the cells with FOXN1 knockdown alone (Fig. 5C). In the Transwell assay,
A549 or H1299 cells co-transfected with shFOXN1 + shEZH2 or shFOXN1 $+\operatorname{sh} \beta$-catenin demonstrated partial alleviation of the effect of FOXN1 knockdown on the NSCLC cell invasive potential (Fig. 5D).

\section{Discussion}

Cell proliferation and metastasis are two important malignant characteristics in NSCLC, which are accompanied by the dynamic changes in the gene expression (13). The results of the present study demonstrated that FOXN1 expression regulated the NSCLC cell growth and invasion. 

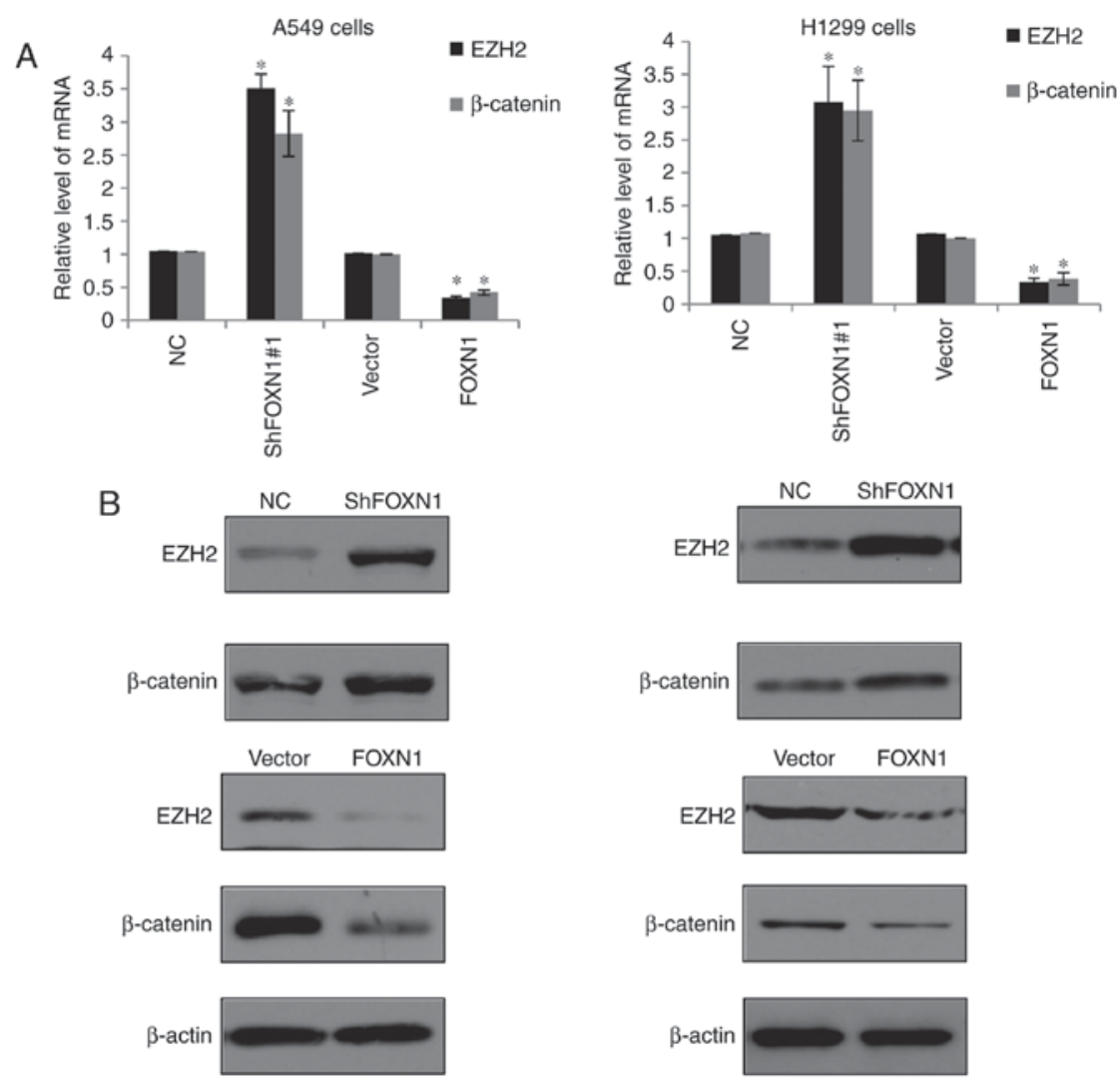

C
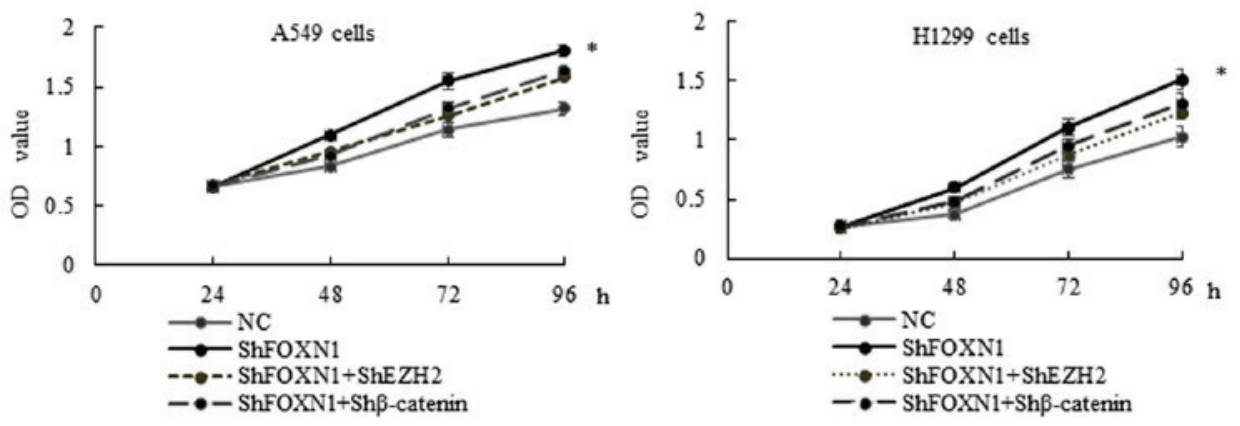

D
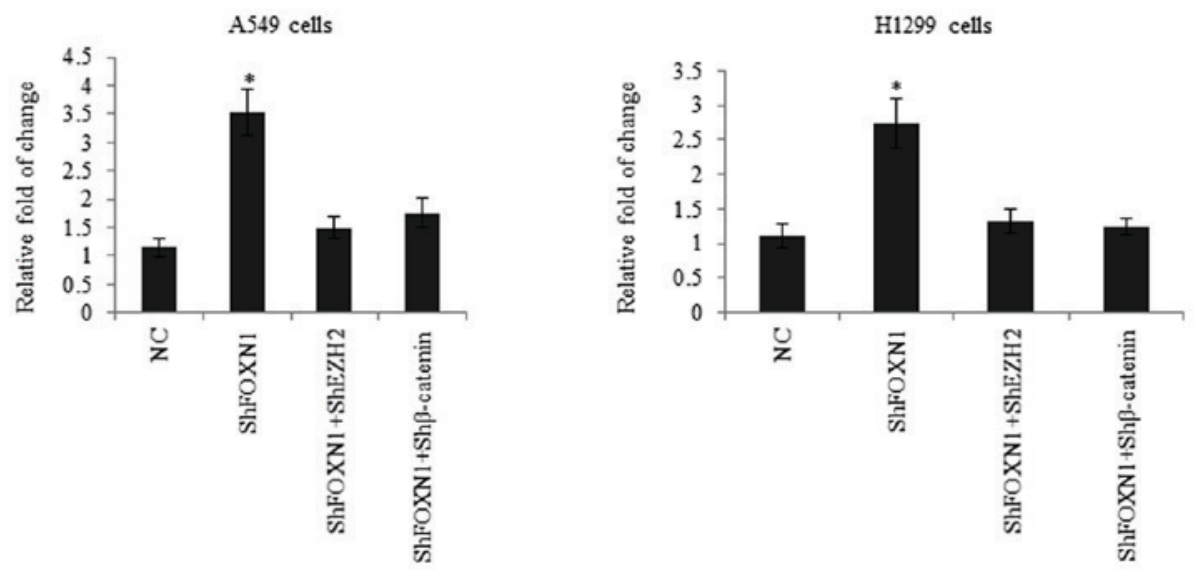

Figure 5. Inhibition of the EZH2/ß-catenin cascade is involved in the suppressive effect of FOXN1 on NSCLC. A549 or H1299 cells were transfected with NC shRNA, shRNA targeting FOXN1, FOXN1 overexpression construct or a control vector. (A) EZH2 and $\beta$-catenin mRNA levels were measured by reverse transcription-quantitative polymerase chain reaction, while (B) the relative protein levels were detected by western blot analysis. (C) MTT and (D) Transwell assays were performed in A549 cells or H1299 cells transfected with NC, shFOXN1, shFOXN1 + shEZH2, or shFOXN1 + sh $\beta$-catenin. Data are represented as the mean \pm standard deviation of three independent experiments. "P $<0.05$ vs. corresponding control group. FOXN1, forkhead box N1; shRNA, short hairpin RNA; NC, negative control shRNA; EZH2, enhancer of zeste homolog 2; OD, optical density. 
Overexpression of FOXN1 could inhibit the NSCLC progression. In the NSCLC patients, higher expression of FOXN1 is associated with better prognosis. To our knowledge, this is the first report about the function of FOXN1 in NSCLC.

The current study further revealed that FOXN1 served major roles in NSCLC invasion by directly targeting EZH2 and $\beta$-catenin therefore suppressing $\mathrm{EZH} 2$ and $\beta$-catenin expression. By epigenetically silencing tumor suppressor genes, EZH2 has been reported to promote carcinogenesis, and overexpression of EZH2 was correlated with NSCLC cell migration (14). Furthermore, increased expression of EZH2 has been correlated with lymph node metastasis, tumor size and TNM stage in NSCLC (15). It has also been reported that the expression of EZH2 was significantly higher in NSCLC brain metastases (16). A previous study reported that inhibition of EZH2 sensitized BRG1 and epidermal growth factor receptor mutant lung tumors to topoisomerase II inhibitors, while knockdown of EZH2 reversed the cisplatin-resistance of NSCLC and gastric cancer cells (17). The regulation of $\mathrm{EZH} 2$ has been revealed by numerous studies in recent years; for instance, microRNA-138 inhibited tumor growth through repression of EZH2 in NSCLC and osteosarcoma cells $(18,19)$. Furthermore, the long non-coding RNA MALAT1 enhanced the oncogenic activities of EZH2 in castration-resistant prostate cancer (20). These aforementioned findings regarding EZH2 were consistent with the observations of the current study. Furthermore, the present results reported that FOXN1 may be a novel regulator of EZH2 in NSCLC. The inhibition of EZH2 by FOXN1 is of importance in the NSCLC suppression.

It has previously been reported that overexpression of $\beta$-catenin was associated with poor overall survival rates in patients with Stage IA-IIA squamous cell lung cancer irrespective of adjuvant chemotherapy (21). By activating the $\beta$-catenin signaling pathway, DEAD-box helicase 5 promoted the proliferation and tumorigenesis of NSCLC (22). In addition, through promoting $\beta$-catenin degradation, Shisa3 was associated with prolonged survival times in lung cancer (23). The majority of these studies focused on the negative regulation of $\beta$-catenin; however, transcriptional repression may also be another important mechanism for $\beta$-catenin inhibition $(24,25)$. The present study reported that FOXN1 was a negative regulator of $\beta$-catenin, thus contributing toward NSCLC repression. Notably, it was observed that FOXN1 served major roles in NSCLC proliferation and invasion by directly targeting EZH 2 and $\beta$-catenin, which suggested that FOXN1 may be a multifunctional regulator in NSCLC.

In conclusion, the present study revealed that FOXN1 inhibited the NSCLC progression and served as a tumor suppressor. EZH2 and $\beta$-catenin may be potential targets for the development of anti-proliferation and anti-invasion in the treatment of NSCLC. However, further investigation is required in order to determine whether the current results may be translated into the development of valuable NSCLC prognosis biomarkers.

\section{Acknowledgements}

Not applicable.

\section{Funding}

No funding was received.

\section{Availability of data and materials}

The datasets used and/or analyzed during the current study are available from the corresponding author on reasonable request.

\section{Authors' contributions}

XJ conceived and designed the work. WW analyzed the data provided technical assistance for the transwell assay. XX and YJ analyzed the data. XJ wrote the paper. All authors read and approved the final manuscript.

\section{Ethics approval and consent to participate}

All human tissues are collected under Institutional Review Committee (IRB) and Health Insurance Portability and Accountability Act (HIPAA) approved protocols.

\section{Consent for publication}

Not applicable.

\section{Competing interests}

The authors declare that they have no competing interests.

\section{References}

1. Jemal A, Siegel R, Ward E, Hao Y, Xu J, Murray T and Thun MJ: Cancer statistics, 2008. CA Cancer J Clin 58: 71-96, 2008.

2. van $\mathrm{Z}$, wijk N and Fong KM: Update in lung cancer: Prologue to a modern review series. Respirology 20: 183-184, 2015.

3. O'Dowd EL and Baldwin DR: Early diagnosis pivotal to survival in lung cancer. Practitioner 258: 21-24, 2-3, 2014.

4. Sharma SP: New therapeutic target for non-small-cell lung cancer. Lancet Oncol 15: e533, 2014.

5. Yang T, Li H, Thakur A, Chen T, Xue J, Li D and Chen M: FOXP4 modulates tumor growth and independently associates with miR-138 in non-small cell lung cancer cells. Tumour Biol 36: 8185-8191, 2015.

6. Cuiffo BG and Karnoub AE: Silencing FOXP2 in breast cancer cells promotes cancer stem cell traits and metastasis. Mol Cell Oncol 3: e1019022, 2015.

7. Feng J, Zhang X, Zhu H, Wang X, Ni S and Huang J: High expression of FoxP1 is associated with improved survival in patients with non-small cell lung cancer. Am J Clin Pathol 138: 230-235, 2012.

8. Liu H, Li W, Yu X, Gao F, Duan Z, Ma X, Tan S, Yuan Y, Liu L, Wang J, et al: EZH2-mediated Puma gene repression regulates non-small cell lung cancer cell proliferation and cisplatin-induced apoptosis. Oncotarget 7: 56338-56354, 2016.

9. Jin J, Zhan P, Katoh M, Kobayashi SS, Phan K, Qian H, Li H, Wang X, Wang X and Song Y; written on behalf of the AME Lung Cancer Collaborative Group: Prognostic significance of $\beta$-catenin expression in patients with non-small cell lung cancer: A meta-analysis. Transl Lung Cancer Res 6: 97-108, 2017.

10. Zell JA, Ignatius Ou SH, Ziogas A and Anton-Culver H: Validation of the proposed International Association for the Study of Lung Cancer non-small cell lung cancer staging system revisions for advanced bronchioloalveolar carcinoma using data from the California Cancer Registry. J Thorac Oncol 2: 1078-1085, 2007.

11. Livak KJ and Schmittgen TD: Analysis of relative gene expression data using real-time quantitative PCR and the 2(-Delta Delta C(T)) method. Methods 25: 402-408, 2001. 
12. Palamaro L, Romano R, Fusco A, Giardino G, Gallo V and Pignata C: FOXN1 in organ development and human diseases. Int Rev Immunol 33: 83-93, 2014.

13. Mittal V: Epithelial mesenchymal transition in aggressive lung cancers. Adv Exp Med Biol 890: 37-56, 2016.

14. Xu C, Hou Z, Zhan P, Zhao W, Chang C, Zou J, Hu H, Zhang Y, Yao X, Yu L and Yan J: EZH2 regulates cancer cell migration through repressing TIMP-3 in non-small cell lung cancer. Med Oncol 30: 713, 2013

15. Geng J, Li X, Zhou Z, Wu CL, Dai M and Bai X: EZH2 promotes tumor progression via regulating VEGF-A/AKT signaling in non-small cell lung cancer. Cancer Lett 359: 275-287, 2015.

16. Behrens C, Solis LM, Lin H, Yuan P, Tang X, Kadara H, Riquelme E, Galindo H, Moran CA, Kalhor N, et al: EZH2 protein expression associates with the early pathogenesis, tumor progression, and prognosis of non-small cell lung carcinoma. Clin Cancer Res 19: 6556-6565, 2013.

17. Zhou W, Wang J, Man WY, Zhang QW and Xu WG: siRNA silencing EZH2 reverses cisplatin-resistance of human non-small cell lung and gastric cancer cells. Asian Pac J Cancer Prev 16: 2425-2430, 2015.

18. Zhu Z, Tang J, Wang J, Duan G, Zhou L and Zhou X: MiR-138 acts as a tumor suppressor by targeting EZH2 and enhances cisplatin-induced apoptosis in osteosarcoma cells. PLoS One 11: e0150026, 2016.

19. Zhang HJ, Zhang H, Zhao M, Lv Z, Zhang X, Qin X, Wang H, Wang S, Su J, Lv X, et al: MiR-138 inhibits tumor growth through repression of EZH2 in non-small cell lung cancer. Cell Physiol Biochem 31: 56-65, 2013.
20. Wang D, Ding L, Wang L, Zhao Y, Sun Z, Karnes RJ, Zhang J and Huang H: LncRNA MALAT1 enhances oncogenic activities of EZH2 in castration-resistant prostate cancer. Oncotarget 6: 41045-41055, 2015

21. Kim Y, Jin D, Lee BB, Cho EY, Han J, Shim YM, Kim HK and Kim DH: Overexpression of $\beta$-catenin and cyclin D1 is associated with poor overall survival in patients with stage IA-IIA squamous cell lung cancer irrespective of adjuvant chemotherapy. J Thorac Oncol 11: 2193-2201, 2016

22. Wang Z, Luo Z, Zhou L, Li X, Jiang T and Fu E: DDX5 promotes proliferation and tumorigenesis of non-small-cell lung cancer cells by activating $\beta$-catenin signaling pathway. Cancer Sci 106: 1303-1312, 2015

23. Chen CC, Chen HY, Su KY, Hong QS, Yan BS, Chen CH, Pan SH, Chang YL, Wang CJ, Hung PF, et al: Shisa3 is associated with prolonged survival through promoting $\beta$-catenin degradation in lung cancer. Am J Respir Crit Care Med 190: 433-444, 2014.

24. Moon RT, Kohn AD, De Ferrari GV and Kaykas A: WNT and beta-catenin signalling: Diseases and therapies. Nat Rev Genet 5: 691-701, 2004.

25. Tseng RC, Lin RK, Wen CK, Tseng C, Hsu HS, Hsu WH and Wang YC: Epigenetic silencing of AXIN2/betaTrCP and deregulation of p53-mediated control lead to wild-type beta-catenin nuclear accumulation in lung tumorigenesis. Oncogene 27: 4488-4496, 2008. 